\title{
THE REDSHIFT OF THE HIGHLY VARIABLE BL LAC OBJECT H0323+022
}

\author{
Alexei V. Filippenko, ${ }^{\text {b),c) }}$ S. DJorgovski, ${ }^{\text {d) }}$ AND Hyron SpINRAD \\ Department of Astronomy, University of California, Berkeley, California 94720 \\ Wallace L. W. SARgent \\ Palomar Observatory, California Institute of Technology, Pasadena, California 91125 \\ Received 7 August 1985; revised 4 September 1985
}

\begin{abstract}
New optical spectra are used to derive the redshift of $\mathrm{H} 0323+022$, a bright, highly variable $\mathrm{x}$-ray source whose nature has been the subject of controversy. Most of the spectra are dominated by a featureless nonstellar continuum, as found in BL Lac objects. Photometric variations of $\gtrsim 2$ mag have been seen over a time interval of only 2 days. The nonstellar light is sometimes faint enough to allow the detection of absorption lines at a redshift $z=0.1471 \pm 0.0005$. These lines are clearly due to stars in the host galaxy, which is easily visible in deep images of the object. The galaxy is probably a relatively normal elliptical with $M_{V} \approx-21$ to -22 , except that a noticeable extension is evident in one quadrant. On 24.15 February 1985 UT, the nonstellar continuum could be described by a power law of index $\alpha \approx 0.6$ (where $f_{v} \propto v^{-\alpha}$ ); its relative strength in the nucleus $\left(2^{\prime \prime} \times 5.2^{\prime \prime}\right)$ was $\sim 62 \%$ at $5460 \AA$, while $M_{V}$ $\approx-22$ to -23 . When the power-law component fades, its slope appears to steepen. There is now no doubt that $\mathrm{H} 0323+022$ is a BL Lac object, rather than a peculiar Galactic star. As discussed by Feigelson et al. (1986), its extremely rapid variability $\left(\Delta L \approx 1 \times 10^{45} \mathrm{erg} \mathrm{s}^{-1}\right.$ in $\left.\Delta t \approx 30 \mathrm{~s}\right)$ is matched only by that of OJ 287 , and the hard $x$ rays must come from a region roughly a factor of $10^{4}$ smaller than that which produces the radio emission.
\end{abstract}

\section{INTRODUCTION}

$\mathrm{H} 0323+022$ is a very rapidly variable $\mathrm{x}$-ray source at Galactic latitude $b=-42.6^{\circ}$, discovered during the $H E A O$ 1 all-sky survey. It is coincident with a stellar object of magnitude $\sim 16$ to 17 on the Palomar Observatory Sky Survey plates. Doxsey et al. (1983) were the first to provide a detailed description of its puzzling properties. An absence of features in the very blue continuum $(U-B=-0.5, B-V=0.5)$, together with the observed $x$-ray and radio characteristics, suggested that $\mathrm{H} 0323+022$ is a BL Lacertae object, but on two occasions the spectrum was seen to exhibit absorption lines apparently like those of G-type stars at zero redshift. Dramatic changes in the $x$-ray flux were measured on time scales of $\lesssim 60 \mathrm{~s}$ and longer.

Margon and Jacoby (1984) argued that H0323 + 022 would be unprecedented in its radio and $x$-ray properties if it were a Galactic object. They obtained a deep, red image of the source and discovered that it is spatially extended on a scale of $\sim 10^{\prime \prime}$. The distance, derived under the assumption that the nebulous component is actually a relatively normal underlying galaxy associated with the bright starlike "nucleus," is in the range $0.5-1.5 \mathrm{Gpc}\left(H_{0}=50 \mathrm{~km} \mathrm{~s}^{-1} \mathrm{Mpc}^{-1}\right.$, $q_{0}=0$ ). If correct, this implies radio, optical, and x-ray luminosities similar to those of most BL Lacs. Spectra of two objects within a few arcminutes of $\mathrm{H} 0323+022$ revealed absorption lines typical of low-velocity $G$ stars; evidently, the conflicting information presented by Doxsey et al. (1983) was due to several unfortunate misidentifications. Margon and Jacoby (1984) concluded that $\mathrm{H} 0323+022$ is almost certainly a new BL Lac object with relatively normal properties, other than its extremely rapid x-ray variability.

a) Based in part on research done at Lick Observatory, University of California.

b) Miller Research Fellow.

${ }^{c}$ Guest Observer at Palomar Observatory, owned and operated by the California Institute of Technology.

d) Present address: Center for Astrophysics, 60 Garden St., Cambridge, MA 02138.
Recently, Feigelson et al. (1986) completed a very thorough investigation of $\mathrm{H} 0323+022$ using a number of techniques. They found strong evidence in support of the BL Lac classification. In particular, $\mathrm{H} 0323+022$ exhibits a steep, featureless optical continuum whose polarization varies between $2 \%$ and $9 \%$, a flat, somewhat polarized radio spectrum, and photometric variability at optical, radio, and $\mathrm{x}$ ray wavelengths. Using an empirical relation between the effective radius of an elliptical galaxy and its absolute magnitude, they estimated a redshift $z=0.13 \pm 0.05$ from measurements of the optical nebulosity in a CCD image of $\mathrm{H} 0323+022$. A similar result was found from comparisons with the apparent magnitudes, angular extents, and redshifts of the host galaxies of 29 quasars. Attempts to detect absorption or emission lines in spectra of the object were unsuccessful, as in previous studies.

Many interpretations of the physical processes in, and the characteristics of, $\mathrm{H} 0323+022$ are critically dependent on its redshift. The estimates made by Margon and Jacoby (1984) and by Feigelson et al. (1986) rely heavily on the assumption of a normal elliptical galaxy as the host, but numerous examples (e.g., Halpern et al. 1986) demonstrate that this may be far from true in individual cases. We therefore decided to directly measure the redshift of $\mathrm{H} 0323+022$ with new optical spectra having very high quality.

\section{OBSERVATIONS AND REDUCTIONS \\ a) Lick Images and Spectra}

Most of the data were obtained with the $3 \mathrm{~m}$ Shane telescope at Lick Observatory (Table I). Initially, two direct images of $\mathrm{H} 0323+022$, each of $100 \mathrm{~s}$ duration, were acquired with a Texas Instruments (TI) CCD at the Cassegrain focus (Lauer et al. 1983; Miller 1983). The Lick $r_{s}$ bandpass (Djorgovski 1985), whose effective wavelength and half-power width are $\sim 6890 \AA$ and $\sim 1600 \AA$, respectively, was used.

Figure 1 illustrates the averaged CCD frame; a contour plot is superposed on the gray-scale image so that the shapes of the outer isophotes may be more readily visualized. Com- 
TABLE I. Journal of observations.

\begin{tabular}{|c|c|c|c|c|c|c|}
\hline UT date & Telescope & $\begin{array}{l}\text { Exp. } \\
(\min )\end{array}$ & $\begin{array}{l}\text { Mode }^{a} / \\
\text { detector }\end{array}$ & $\begin{array}{l}\text { Aper. or } \\
\text { slit(" } \times \text { ") }\end{array}$ & $\begin{array}{l}\text { P.A. } \\
\text { (deg) }\end{array}$ & $\Delta \lambda(\AA)$ \\
\hline $\begin{array}{l}21 \text { Dec. } 1984 \\
29 \text { Dec. } 1984 \\
25 \text { Jan. } 1985 \\
21 \text { Feb. } 1985 \\
22 \text { Feb. } 1985 \\
23 \text { Feb. } 1985 \\
24 \text { Feb. } 1985\end{array}$ & $\begin{array}{l}\text { Shane } \\
\text { Shane } \\
\text { Shane } \\
\text { Shane } \\
\text { Shane } \\
\text { Shane } \\
\text { Hale }\end{array}$ & $\begin{array}{l}3.3 \\
91 \\
128 \\
112 \\
80 \\
50 \\
60\end{array}$ & $\begin{array}{l}\text { D/CCD } \\
\text { S/CCD } \\
\text { S/ITS } \\
\text { S/ITS } \\
\text { S/ITS } \\
\text { S/CCD } \\
\text { S/CCD }\end{array}$ & $\begin{array}{c}120 \times 120^{\mathrm{b}} \\
2 \times 120 \\
4 \times 4 \\
4 \times 4 \\
4 \times 4 \\
2 \times 120 \\
2 \times 100\end{array}$ & $\begin{array}{l}90 \\
60 \\
90 \\
90 \\
90 \\
92 \\
43\end{array}$ & $\begin{array}{l}6100-7700^{c} \\
4100-8000 \\
3110-5370 \\
3200-5490 \\
3240-5530 \\
4100-8000 \\
4250-5120 \\
6210-6860\end{array}$ \\
\hline 25 Feb. 1985 & Shane & 33 & $\mathrm{~S} / \mathrm{CCD}$ & $2 \times 120$ & 90 & $4100-6700$ \\
\hline
\end{tabular}

$\mathrm{D}$ and $\mathrm{S}$ refer to direct imaging and spectroscopy, respectively.

b Approximate field of view, rather than size of slit or aperture.

${ }^{c}$ Approximate half-power bandpass, rather than spectral range.

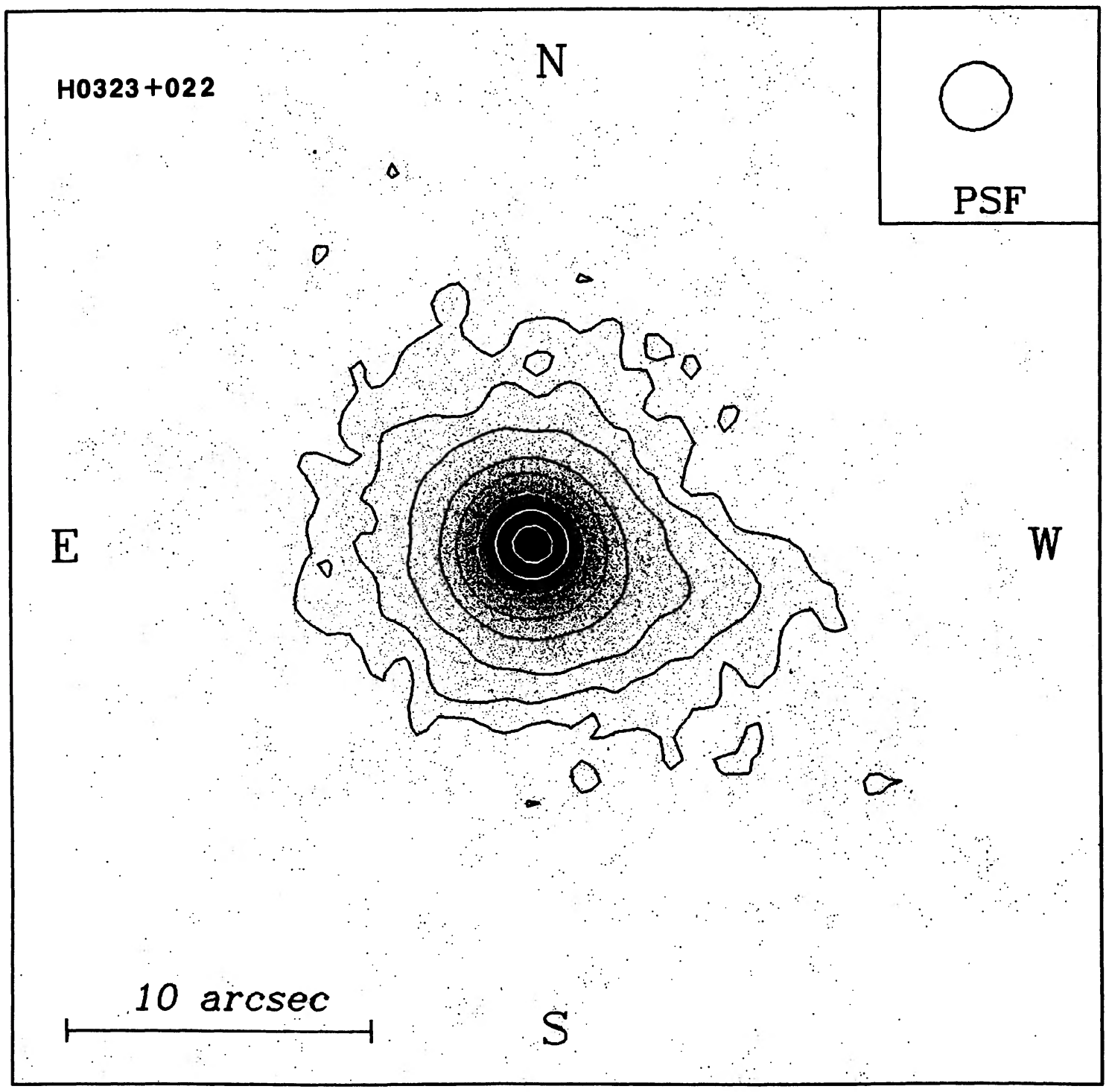

FIG. 1. Gray scale and contour representation of a red $\left(\lambda_{\text {eff }} \approx 6890 \AA\right)$ CCD image of H0323 + 022, obtained at Lick Observatory on the night of 21 December 1984 UT. The contour levels are at $0.5 \%, 1 \%, 2 \%, 5 \%, 10 \%, 20 \%, 50 \%$, and $80 \%$ of the peak intensity. A $50 \%$ contour of a nearby field star serves to define the point-spread function (PSF, upper right). 
parison with the point-spread function of the system, as defined by a nearby field star, clearly shows that $\mathrm{H} 0323+022$ is extended, with a maximum diameter of $\sim 20^{\prime \prime}$. As noted by Margon and Jacoby (1984) and by Feigelson et al. (1986), the greatest extension occurs in the southwest quadrant, at a position angle (P.A.) of $\sim 240^{\circ}$. The nature of this asymmetric portion is not yet known, but it is probably not a companion galaxy since there is evidence of other, smaller irregularities elsewhere among the contour lines. Our azimuthally averaged intensity profile of $\mathrm{H0323}+022$ is similar to that shown by Feigelson et al.(1986).

Spectra were obtained with several different detectors, in some cases as part of an international effort to observe the object simultaneously over a wide range of wavelengths (Urry 1985). The Lick CCD system employs a long slit as an entrance aperture for the spectrograph, so the nucleus and extended fuzz were analyzed separately in the two-dimensional (2-D) data. The spectral resolution, defined by the full width at half-maximum (FWHM) of unblended emission lines from the night sky, was typically $\sim 15 \AA$. Division by the spectrum of an intrinsically featureless quartz lamp adequately removed pixel-to-pixel differences in the sensitivity of the detector.

An image-tube scanner (ITS; Robinson and Wampler 1972; Miller, Robinson, and Wampler 1976) was used at Lick to obtain one-dimensional (1-D) spectra of $\mathrm{H} 0323+022$ at the blue end $(\sim \lambda \lambda 3110-5530)$. One of the two 4 " $\times 4$ " apertures was always centered on the nucleus, while the other sampled the background sky $35^{\prime \prime}$ to the east and west. As with the Lick CCD data, standard stars from the lists of Oke (1974) and Stone (1977) were used to calibrate the spectral response. In most cases, however, the presence of cirrus clouds prevented measurement of absolute fluxes. Using a grating with 600 lines $\mathrm{mm}^{-1}$, we achieved a spectral resolution of $\sim 10 \AA$.

The Lick data were reduced according to the methods described by Djorgovski and Spinrad (1983; 2-D) and by Osterbrock (1977; 1-D). We found several redshift candidates, based on possible absorption and/or emission lines, but a single unambiguous value could not be derived. Although in retrospect one of these candidates turned out to be correct, the observations were generally of too low resolution for confident detection of weak features. Furthermore, the ITS often produces spurious "spikes" and other noise that is not governed by Poisson statistics.

\section{b) Palomar Spectra}

On 24 February 1985 UT, we used the double spectrograph (Oke and Gunn 1982) attached to the Cassegrain focus of the Hale $5 \mathrm{~m}$ telescope at Palomar Observatory. A long slit of $2^{\prime \prime}$ width was placed across the nucleus at P.A. $=43^{\circ}$, to minimize light losses caused by atmospheric dispersion (Filippenko 1982) at the moderately high airmass of $\sec z \approx 1.5$. Note that this is not far from the P.A. of the extended nebulosity in Fig. 1. The integration time was $1 \mathrm{hr}$. Conditions were photometric, and the seeing was $1.5^{\prime \prime}-2$ " (FWHM). Bright standard stars from the list of Oke and Gunn (1983) were observed with slit widths of 2 " and 10" to ensure accurate flux calibration. Detectors consisted of two TI CCDs, each with $800 \times 800$ square pixels $\left(0.58^{\prime \prime}\right.$ pixel $^{-1}$, red; $0.40^{\prime \prime}$ pixel $^{-1}$, blue). Reduction procedures were similar to those described by Filippenko and Sargent (1985a).

One-dimensional spectra, covering the central 9 and 13 pixels of the red and blue CCDs (respectively), were extracted for both $\mathrm{H} 0323+022$ and the standard stars, so the effective entrance aperture was $2^{\prime \prime} \times 5.2^{\prime \prime}$ in all cases. Since observing conditions were nearly constant, we estimate that the derived brightness of the nucleus of $\mathrm{H} 0323+022$, $m(\lambda 5000)=17.4$ (see Sec. III), is uncertain by only $\sim \pm 0.2$ mag. Of course, this measurement does not include much of the light from the extended nebulosity, and is therefore a lower limit to the apparent magnitude of the entire object.

\section{RESULTS \\ a) Redshift}

Figure 2 illustrates the blue Palomar spectrum of $\mathrm{H} 0323+022$ on the magnitude scale defined by Oke and Gunn (1983). The original data, whose resolution (FWHM) is $\sim 4.3 \AA$, were smoothed with a Gaussian of similar width

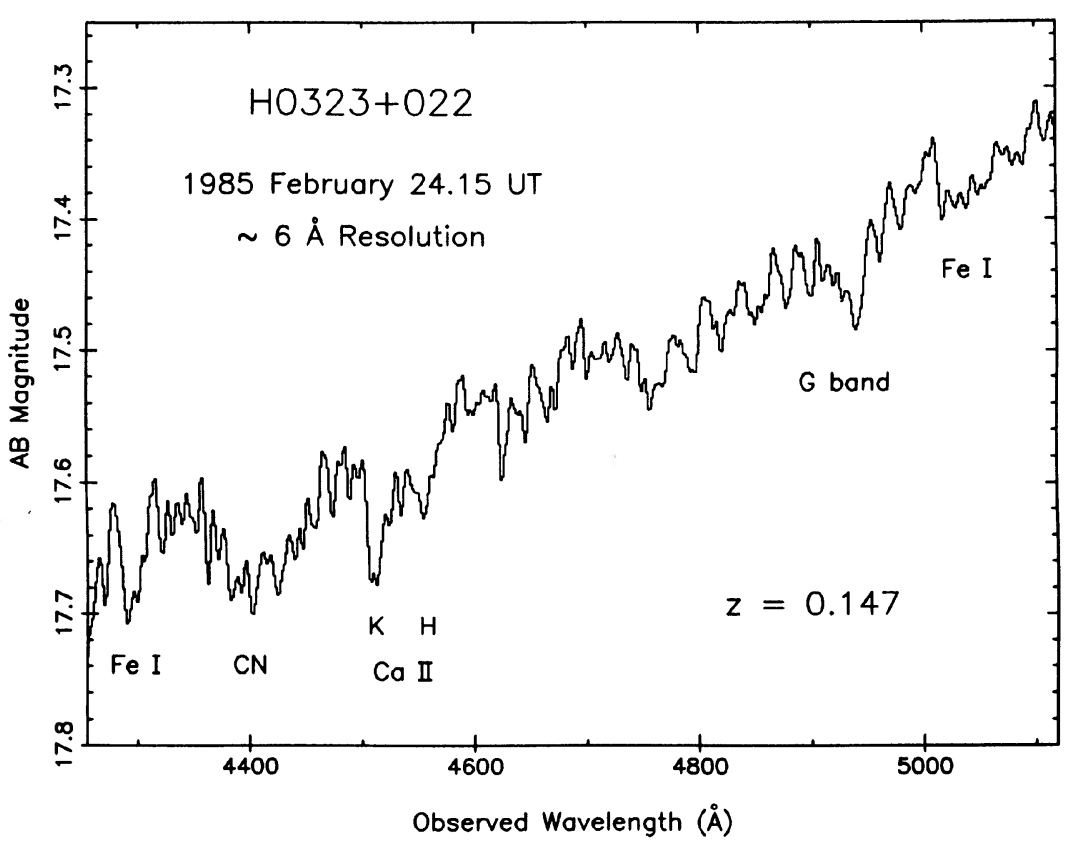

FIG. 2. Slightly smoothed, blue spectrum of $\mathrm{H} 0323+022$, obtained with the Hale telescope at Palomar Observatory. The effective entrance aperture was $2^{\prime \prime} \times 5.2^{\prime \prime}$. Magnitude $A B$ $=-2.5 \log \left(f_{v}\right)-48.6$, where $f_{v}$ is in $\operatorname{erg~s}^{-1} \mathrm{~cm}^{-2} \mathrm{~Hz}^{-1}$. Prominent absorption lines from an old stellar population are visible at a redshift of $0.1471 \pm 0.0005$ 
to yield a final resolution of $\sim 6 \AA$. Absorption lines of Ca II, $\mathrm{Fe} \mathrm{I}$, and other species are clearly visible; as first reported by Filippenko and Sargent (1985b), the observed wavelengths are consistent with a redshift $z=0.147$ for the starlight and, presumably, for the BL Lac nucleus itself.

This identification is supported by the red spectrum in Fig. 3, which has also been smoothed with a Gaussian whose FWHM is comparable to the original resolution $(\sim 2.2 \AA)$. The $\mathrm{Na}$ I $\mathrm{D}$ doublet is unmistakably present at the expected position. Analysis of all the strongest absorption lines in both spectra leads to a final value of $0.1471 \pm 0.0005$ for the redshift of $\mathrm{H} 0323+022$. Note that the number calculated by Feigelson et al. (1986) from the apparent size of the optical nebulosity, $z=0.13 \pm 0.05$, is remarkably close to the true redshift. Margon and Jacoby (1984) made a similar estimate one year earlier, and came to essentially the same conclusion. The assumption that the underlying galaxy is a relatively normal elliptical with $M_{V} \approx-21$ to -22 is therefore probably correct.

It is perhaps surprising that the redshift was determined so easily from the Palomar data, considering how much time was spent on this object at Lick Observatory, but the moderately high resolution of the Palomar spectra simplified the detection of weak stellar absorption lines. Moreover, the nonstellar continuum of $\mathrm{H0323}+022$ was relatively faint during the Palomar observations (24 February 1985 UT). In fact, very rough spectrophotometry with the Lick CCD indicates that the nucleus faded by $\sim 0.5 \mathrm{mag}$ in $B, \sim 0.2 \mathrm{mag}$ in $V$, and $\sim 0.1 \mathrm{mag}$ in $R$ from 29 December 1984 to 23 February $1985 \mathrm{UT}$, and subsequently faded by as much as $\sim 2.7$ mag in $B$ and $\sim 2$ mag in $V$ the following two nights! Such variations are somewhat larger than those reported by Feigelson et al. (1986), but the uncertainties in our measurements are substantial ( $\gtrsim \pm 0.5 \mathrm{mag}$ ).

\section{b) Stellar and Nonstellar Continua}

A quantitative estimate of the nonstellar continuum in $\mathrm{H} 0323+022$ was made by using least-squares-fitting tech- niques to decompose the Palomar spectra into a power law of the form $f_{v} \propto v^{-\alpha}$ and a "standard" giant elliptical galaxy (Yee and Oke 1978). After converting the spectra to rest wavelengths and heavily smoothing with a Gaussian (FWHM $=40 \AA$ ), the procedure described by Halpern and Filippenko (1984) was followed. The Galactic reddening of $E_{B-V} \approx 0.07 \mathrm{mag}$ (Burstein and Heiles 1982) was removed, while reddening in $\mathrm{H0323}+022$ itself was assumed to be negligible. Since only two relatively small wavelength ranges were available in the decomposition, we restricted ourselves to two free parameters: the fractional contribution of the power law at $5460 \AA$, and its spectral index $(\alpha)$. This method is sensitive to the slope of the spectra and to certain broad features such as the large "break" near $4000 \AA$, but not to the strength of narrow absorption lines. Potential sources of error include a relative uncertainty of $\leqslant 0.1 \mathrm{mag}$ between the absolute calibration of the red and blue CCD spectra, unknown dust in $\mathrm{H} 0323+022$, and the possibility that the underlying galaxy is not well represented by the spectrum of an average giant elliptical.

The results are illustrated in Fig. 4. A reasonably good fit was achieved with $\alpha=0.6$; this, however, is actually a compromise between the best "formal" value found by the computer program, and the value that leads to the closest overall agreement between equivalent widths of absorption lines in the remaining galaxy and in typical elliptical galaxies. A much steeper slope, for example, makes the power law completely dominate the fit, so that the equivalent widths of absorption lines are too large after the featureless continuum is subtracted from the original, unsmoothed data. Too flat a slope, on the other hand, leads to a relatively small contribution from the nonstellar continuum, and the observed equivalent widths are inconsistent with this.

Of course, it is not possible to determine $\alpha$ with high precision because of the very limited spectral coverage, but an uncertainty of \pm 0.5 seems reasonable. A slope steeper than 1.5 is completely excluded, since the dereddened data can be adequately represented purely by the power law $f_{v} \propto v^{-1.5}$, with no stellar component. (The slope is 1.8 if the Galactic

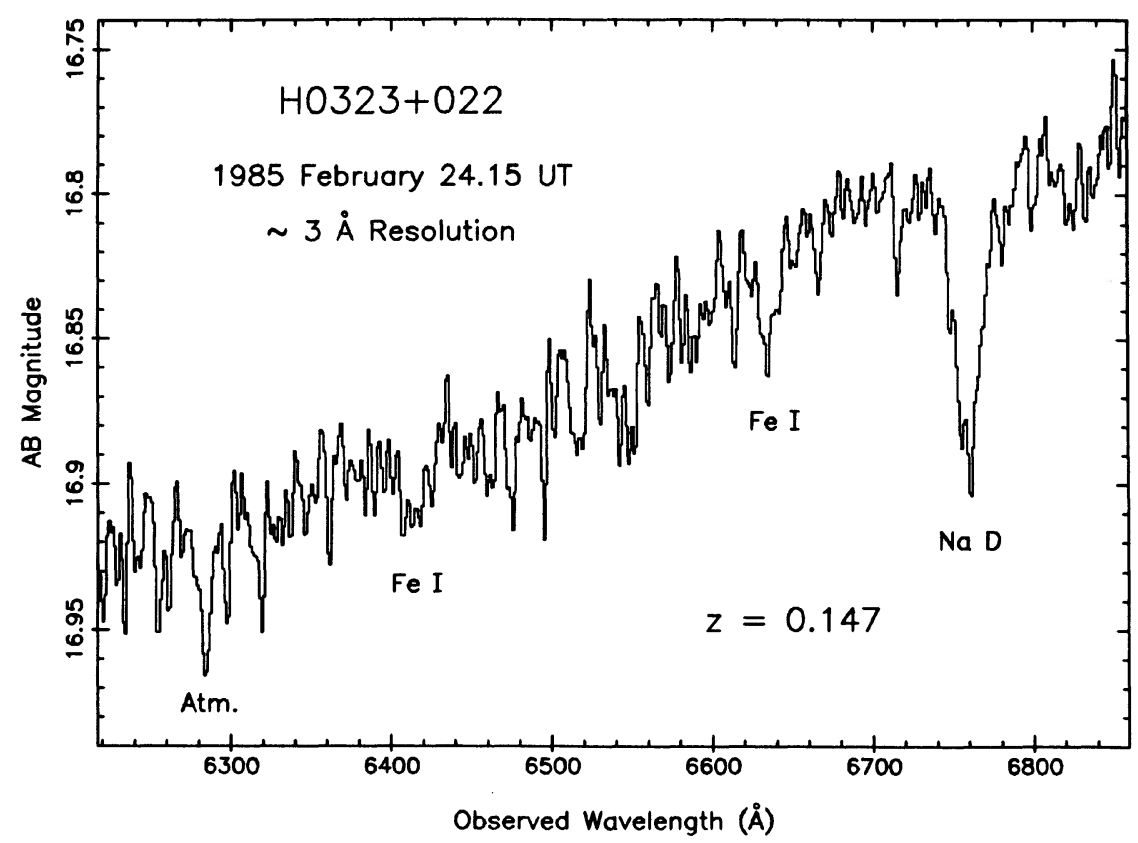

FIG. 3. Slightly smoothed, red spectrum of $\mathrm{H} 0323+022$, obtained with the Hale telescope at Palomar Observatory. Strong Na I D absorption is visible at $\sim 6758 \AA$, confirming the redshift derived in Fig. 2. "Atm." refers to telluric $\mathrm{O}_{2}$ absorption. 
H0323+022: Power Law + Standard Galaxy Decomposition

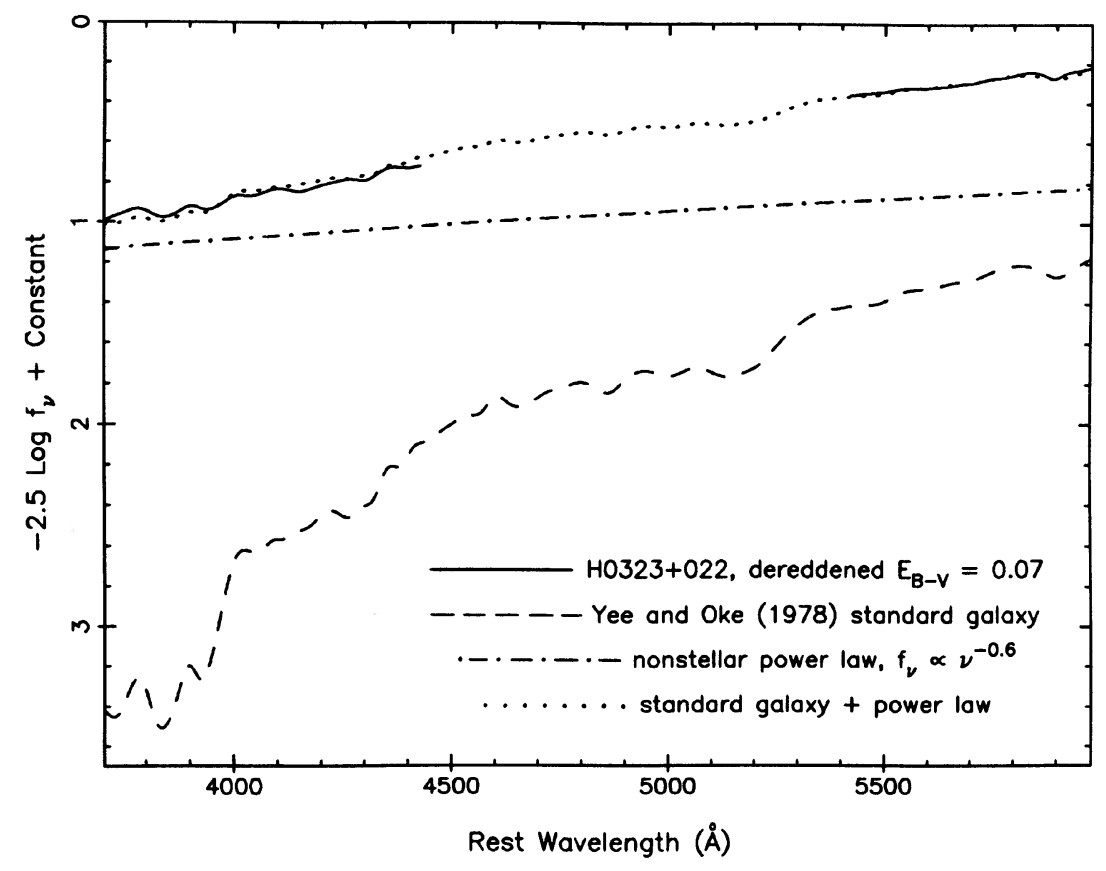

FIG. 4. The heavily smoothed continuum of $\mathrm{H} 0323+022$ (solid line) is decomposed into a nonstellar power law (dot-dashed line) of index 0.6 and a "standard" giant elliptical galaxy (dashed line); a dotted line represents the best least-squares fit. Data from Figs. 2 and 3 are used in the analysis, but the redshift $(z=0.1471)$ and Galactic reddening $\left(E_{B-V}\right.$ $=0.07 \mathrm{mag}$ ) have been removed. Each numbered tick mark on the ordinate scale corresponds to one magnitude.

reddening of $E_{B-V} \approx 0.07 \mathrm{mag}$ is ignored in the decomposition.) Conversely, $\alpha \leqslant 0$ is ruled out strongly because it makes the underlying galaxy much too prominent. Analysis of the Lick spectra suggests that the spectral index became steeper by more than one unit when $\mathrm{H} 0323+022$ faded by $\gtrsim 2$ mag during a 2 day interval in late February 1985 . Several other BL Lac objects are known to exhibit similar behavior (Stein, O'Dell, and Strittmatter 1976), although some of the color variations reported by Feigelson et al. (1986) are quite peculiar.

The decomposition in Fig. 4 shows that with $\alpha=0.6$ the power law is over 2 mag brighter than the galaxy at $\sim 3700$ $\AA$, whereas at $\gtrsim 5460 \AA$ it contributes only $\sim 62 \%$ of the light. Thus, the equivalent width of $\mathrm{Na} \mathrm{D}(1.5 \pm 0.4 \AA)$ in the original spectrum is consistent with a normal strength of $\sim 4$ $\AA ̊$ (e.g., Tonry and Davis 1981; Malkan and Filippenko 1983) diluted by the power law.

The measured magnitude of $\mathrm{H} 0323+022$ through an entrance aperture of $2^{\prime \prime} \times 5.2^{\prime \prime}$ is $m(\lambda 5000)=17.4 \pm 0.2 \mathrm{mag}$ (Fig. 2); simple extrapolation yields $m(\lambda 5460)=17.2 \pm 0.3$ mag. From Fig. 4, we see that at $\sim 4760 \AA$, which is the corresponding rest wavelength, the standard galaxy is $\sim 1.2$ mag fainter than the total. Thus, the underlying galaxy has a visual magnitude of $m_{V} \approx 18.4 \pm 0.4$ through a $2^{\prime \prime} \times 5.2^{\prime \prime}$ aperture, or $\sim 18.2$ if Galactic extinction is removed. Considering the extent of the nebulosity in Fig. 1, this compares favorably with the overall value of $\sim 17.5$ (or $\sim 17.3$ if $A_{V}=0.2$ mag) obtained by Feigelson et al. (1986) from their CCD image. Using $q_{0}=0, z=0.1471$, and $m_{V}=17.3$, we find that $M_{V} \approx-21$ to -22 for the host galaxy, depending on whether $H_{0}=75$ or $50 \mathrm{~km} \mathrm{~s}^{-1} \mathrm{Mpc}^{-1}$. This is roughly 1-2 mag fainter than the average first-ranked elliptical galaxy in clusters (e.g., Sandage 1976; Bahcall 1977), and it is comparable to the characteristic magnitude $M$. in the luminosity function of galaxies (Schechter 1976). The absolute magnitude of the nonstellar component on 24.15 February 1985 was $\sim-22$ to -23 , close to that of faint QSOs (e.g., Hazard 1979), but it is often more luminous than this.

Figure 5 illustrates the blue Palomar spectrum of $\mathrm{H} 0323+022$ in the object's rest frame, after subtraction of the power-law continuum. Galactic reddening was not removed. IC 4889, a bright southern S0 galaxy, is shown for comparison. The overall shape of the continuum, as well as the individual absorption lines and their equivalent widths, are closely matched in the two objects, adding further evidence that the host galaxy of the BL Lac is a relatively normal elliptical or early-type spiral. In particular, strong Balmer lines are absent, and the $\mathrm{CN}$ band at $\sim 3840 \AA$ is very prominent, so a young stellar population is probably negligible. With data of higher resolution, it would be possible to measure the stellar velocity dispersion and obtain an independent estimate of the absolute luminosity of the galaxy (Faber and Jackson 1976).

\section{DISCUSSION AND CONCLUSIONS}

We have used new, high-quality spectra to obtain an absorption-line redshift of $0.1471 \pm 0.0005$ for $\mathrm{H} 0323+022$, an object that exhibits extremely rapid variability at x-ray energies. This confirms the belief of Margon and Jacoby (1984) that $\mathrm{H0323}+022$ is extragalactic, and strongly supports their classification as a new BL Lac object. There are no emission lines visible, and the absorption lines are typical of an old stellar population in a normal elliptical galaxy or in the bulge of an early-type spiral. Variations of $\gtrsim 2 \pm 0.5 \mathrm{mag}$ have been detected in the optical spectra over a period of only 2 days.

The optical continuum, as measured at Palomar Observatory on 24.15 February 1985 UT, has been decomposed into a featureless power law and a standard elliptical galaxy; the derived spectral index, $\alpha=0.6$, is flatter than that found in most radio-selected BL Lacs $(\alpha \approx 2$; Stein, O'Dell, and Strittmatter 1976). X-ray selected BL Lacs, on the other hand, 


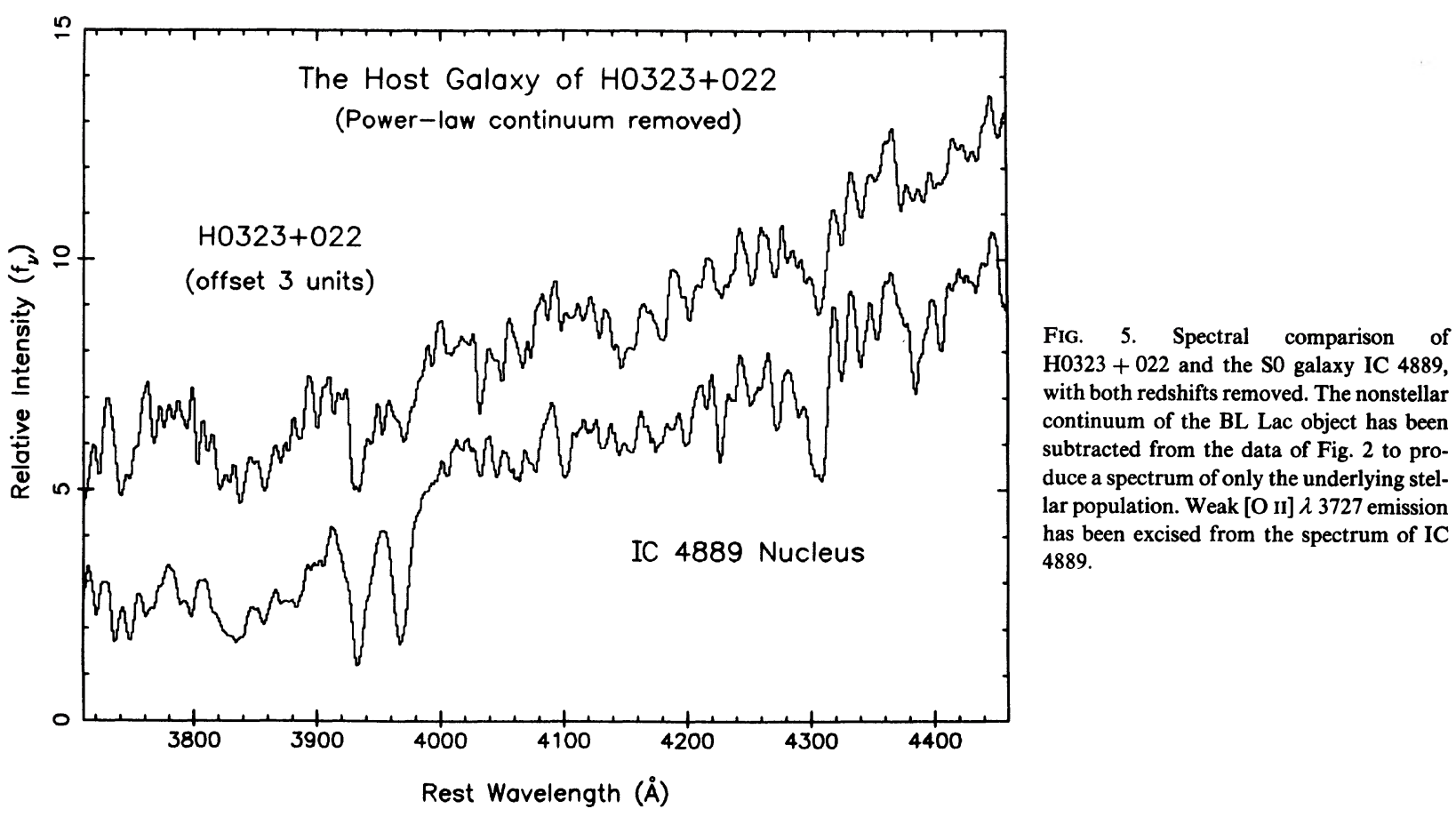

typically have $\alpha \approx 1$ (Stocke et al. 1982), closer to the value found here. Similarly, Halpern et al. (1986) show that the slope is $\sim 0.5$ in $1 \mathrm{E} 1415.6+2557$, another $\mathrm{x}$-ray selected BL Lac object. As mentioned by these authors, it is possible that the frequency of the synchrotron break is above the optical window, whereas it is below in radio-selected BL Lacs having $\alpha \approx 2$. X-ray selection favors objects in which the synchrotron break is at the highest frequencies, and it is not surprising that they have relatively flat optical slopes. It is interesting to note, however, that we saw the spectral index become considerably steeper as the nonstellar component of $\mathrm{H} 0323+022$ faded.

The spectra presented in this paper show that the redshift estimates made by Margon and Jacoby (1984) and by Feigelson et al. (1986) are very close to the correct value. Since $z=0.15$ was assumed throughout the analysis of Feigelson et al., their conclusions need not be modified in this respect. In particular, large flux variations $\left(\Delta L \approx 1 \times 10^{45} \mathrm{erg} \mathrm{s}^{-1}\right)$ during short time intervals $(\Delta t \approx 30 \mathrm{~s})$ imply that the $\mathrm{x}$ rays come from a compact region $\sim 10^{12} \delta \mathrm{cm}$ in size (where $\delta$ is the relativistic Doppler beaming factor). An important con- sequence is that the hard $\mathrm{x}$ rays and the polarized component of the optical continuum must come from a region that is roughly four orders of magnitude smaller than that which produces the radio and unpolarized optical radiation. Thus, the simple synchrotron self-Compton model often used to explain $\mathrm{x}$-ray emission in BL Lac objects cannot be applied to $\mathrm{H} 0323+022$.

We thank M. Doyle, J. Henning, S. Staples, and D. Tennant for their assistance at Palomar Observatory, as well as B. Alcott, K. Baker, C. Clark, W. Earthman, J. Morey, and M. Owens at Lick Observatory. Dr. H. E. Smith and R. P. S. Stone provided useful information for operating the Lick ITS. Drs. H. Bradt and C. M. Urry aroused our initial interest in $\mathrm{H} 0323+022$, and Dr. W. Romanishin communicated results prior to publication. Valuable conversations with $\mathbf{R}$. M. Rich and Dr. J. P. Halpern are gratefully acknowledged. This work was supported by the Miller Institute for Basic Research in Science (A. V. F.), a University of California Regents Fellowship (S.D.), NSF Grant No. AST 84-01788 (H.S.), and NSF Grant No. AST 84-16704 (W.L.W.S).

\section{REFERENCES}

Bahcall, N. A. (1977). Annu. Rev. Astron. Astrophys. 15, 505.

Burstein, D., and Heiles, C. (1982). Astron. J. 87, 1165.

Djorgovski, S. (1985). Publ. Astron. Soc. Pac. (in press).

Djorgovski, S., and Spinrad, H. (1983). In Proceedings of the AAS /OSA

Joint Topical Meeting on Information Processing in Astronomy and Optics (Optical Society of America, Washington, DC), p. ThB2-1.

Doxsey, R., Bradt, H., McClintock, J., Petro, L., Remillard, R., Ricker, G.,

Schwartz, D., and Wood, K. (1983). Astrophys. J. Lett. 264, L43.

Faber, S. M., and Jackson, R. E. (1976). Astrophys. J. 204, 668.

Feigelson, E. D., et al. (1986). Astrophys. J. (in press).

Filippenko, A. V. (1982). Publ. Astron. Soc. Pac. 94, 715.

Filippenko, A. V., and Sargent, W. L. W. (1985a). Astrophys. J. Suppl. 57, 503.

Filippenko, A. V., and Sargent, W. L. W. (1985b). IAU Circ. No. 4059.
Halpern, J. P., and Filippenko, A. V. (1984). Astrophys. J. 285, 475. Halpern, J. P., Impey, C. D., Bothun, G. D., Tapia, S., Skillman, E.D., Wilson, A. S., and Meurs, E. J. A. (1986). Astrophys. J. (in press).

Hazard, C. (1979). In Active Galactic Nuclei, edited by C. Hazard and S. Mitton (Cambridge University, Cambridge), p. 1.

Lauer, T. R., Miller, J. S., Osborne, C. S., Robinson, L. B., and Stover, R. C. (1983). Proc. Soc. Photo-Opt. Instrum. Eng. 445, 132.

Malkan, M. A., and Filippenko, A. V. (1983). Astrophys. J. 275, 477.

Margon, B., and Jacoby, G. H. (1984). Astrophys. J. Lett. 286, L31.

Miller, J. S. (1983). The Lick 3-m Cassegrain CCD Spectrograph (Lick Observatory, University of California, Santa Cruz).

Miller, J. S., Robinson, L. B., and Wampler, E. J. (1976). In Advances in Electronics and Electron Physics (Academic, New York), Chap. 40B, p. 693. 
Oke, J. B. (1974). Astrophys. J. Suppl. 27, 21.

Oke, J. B., and Gunn, J. E. (1982). Publ. Astron. Soc. Pac. 94, 586.

Oke, J. B., and Gunn, J. E. (1983). Astrophys. J. 266, 713.

Osterbrock, D. E. (1977). Astrophys. J. 215, 733.

Robinson, L. B., and Wampler, E. J. (1972). Publ. Astron. Soc. Pac. 84, 161. Sandage, A. (1976). Astrophys. J. 205, 6.

Schechter, P. (1976). Astrophys. J. 203, 297.

Stein, W. A., O'Dell, S. L., and Strittmatter, P. A. (1976). Annu. Rev. Astron. Astrophys. 14, 173.
Stocke, J., Liebert, J., Stockman, J., Danziger, J., Lub, J., Maccacaro, T., Griffiths, R., and Giommi, P. (1982). Mon. Not. R. Astron. Soc. 200, 27P.

Stone, R. P. S. (1977). Astrophys. J. 218, 767.

Tonry, J. L., and Davis, M. (1981). Astrophys. J. 246, 666.

Urry, C. M. (1985). Private communication.

Yee, H. K. C., and Oke, J. B. (1978). Astrophys. J. 226, 753. 